\title{
Factors associated with blood culture positivity in patients with complicated skin and skin structure infection -a population-based study
}

\author{
Mika Halavaara $^{1}$ - liro H. Jääskeläinen ${ }^{1}$ - Lars Hagberg ${ }^{2}$. Asko Järvinen ${ }^{1}$
}

Received: 6 February 2019 / Accepted: 5 April 2019 / Published online: 22 April 2019

(C) The Author(s) 2019

\begin{abstract}
Skin and skin structure infection (SSSI) is classified as complicated (cSSSI) if it involves deep subcutaneous tissue or requires surgery. Factors associated with blood culture sampling and bacteremia have not been established in patients with cSSSI. Moreover, the benefit of information acquired from positive blood culture is unknown. The aim of this study was to address these important issues. In this retrospective population-based study from two Nordic cities, a total of 460 patients with cSSSI were included. Blood cultures were drawn from $258(56.1 \%)$ patients and they were positive in 61 (23.6\%) of them. Factors found to be associated with more blood culture sampling in multivariate analysis were diabetes, duration of symptoms shorter than 2 days and higher C-reactive protein (CRP) level. Whereas factors associated with less frequent blood culture sampling were peripheral vascular disease and a surgical wound infection. In patients from whom blood cultures were taken, alcohol abuse was the only factor associated with culture positivity, as CRP level was not. Patients with a positive blood culture had antibiotic streamlining more often than non-bacteremic patients. A high rate of blood culture positivity in patients with cSSSI was observed. Factors related to more frequent blood culture sampling were different from those associated with a positive culture.
\end{abstract}

Keywords Skin and soft tissue infection $\cdot$ Cellulitis $\cdot$ C-reactive protein $\cdot$ Bacteremia $\cdot$ Abscess $\cdot$ Bloodstream infection

\section{Introduction}

Skin and skin structure infections (SSSIs) are among the most common bacterial infections in patients presenting in emergency rooms and their incidence is rising [1-3]. In 1998, FDA classified SSSI as complicated (cSSSI) if it involves deep subcutaneous tissues or requires surgery [4]. Although initially designed for the clinical trials, the umbrella term cSSSI is still useful in the detection of the most severe forms of SSSIs [5].

Blood cultures are not routinely recommended for patients with SSSI $[6,7]$. This is mainly because positive findings have

Mika Halavaara

mika.halavaara@hus.fi

1 Department of Infectious Diseases, Inflammation Center, Helsinki University Hospital and University of Helsinki, P.O. Box 372, 00029 HUS Helsinki, Finland

2 Department of Infectious Diseases, Institute of Biomedicine, Sahlgrenska Academy, University of Gothenburg,

Gothenburg, Sweden been rare [8] and have only seldom affected antibiotic treatment [9]. However, this might not be generalizable to all SSSIs since the rate of bacteremia has been reported to increase in more severe cases. Whereas blood culture positivity of $4.6 \%$ has been reported in erysipelas, it was $7.9 \%$ in cellulitis [10] and even higher rate of $11.9 \%$ was reported in a recent European survey on cSSSI [11]. Factors associated with bacteremia in cSSSI have not been studied, but in less severe SSSI comorbidity [12] in another study, up to 11 patient factors, including male gender and older age, were linked to bacteremia [13].

We conducted a population-based study including $460 \mathrm{pa}-$ tients with cSSSI from two Nordic cities and reported that at least $13.3 \%$ of patients had a bloodstream infection with equal yield of one fourth of samples being positive in both study sites [14]. Male gender and cellulitis were associated with blood culture sampling and bacteremia with later clinical stability $[14,15]$. In the present study, we analysed further from the same real-life setup factors predicting and associated with blood culture positivity and how the knowledge of blood culture positivity affected the treatment. 


\section{Materials and methods}

The study design was a retrospective observational cohort study. All adult patients hospitalised for cSSSI within a 4year period 2008-2011 in Helsinki University Hospital and Helsinki City Hospital in Helsinki, Finland (604,000 inhabitants), and Sahlgrenska University Hospital in Gothenburg, Sweden $(525,000$ inhabitants), were included in the study [14]. Patients were recognised using International Classification of Diseases (ICD-10) codes and demographic and clinical data were collected from the medical records. These hospitals have the only emergency departments in their catchment areas why virtually all hospitalised patients with cSSSI have been included enabling the population-based approach. The prevalence of methicillin-resistant Staphylococcus aureus was 2.8\% in Finland [16] and 0.8\% in Sweden in 2011 [17].

Detailed study protocol is presented in the primary publication of this study [14]. In short, to be included, patients were required to have infection affecting deeper soft tissue (e.g. cellulitis or fasciitis), infection requiring significant surgical intervention, infection which developed on a lower extremity in a patient with diabetes mellitus or peripheral vascular disease or to have a major abscess or an infected ulcer. Patients also had to have at least one systemic sign of infection: temperature $>38{ }^{\circ} \mathrm{C}$ or $<36{ }^{\circ} \mathrm{C}$ or white blood cell count $>$ $10,000 / \mathrm{mm}^{3}$ or $<4000 / \mathrm{mm}^{3}$.

\section{Study definitions and statistical analyses}

Microbial diagnosis was obtained by blood culture, bacterial culture of tissue or superficial swabs in routine cultures. Cellulitis/fasciitis was defined as an infection without abscess, diabetic foot/leg ulcer or peripheral vascular disease ulcer. The evaluation of clinical stability was based on improvement of vital signs and decrease of fever. Streamlining was defined as change of antibiotic therapy to pathogen specific one. Microbes from normal cutaneous flora (e.g. coagulasenegative staphylococci) were generally not considered as pathogens in blood cultures and an infectious disease specialist assessed each case. Antibiotic treatment prescribed before admission or before the fulfilment of cSSSI criteria during the hospitalisation was recorded.

Categorical variables were summarised using counts and percentages. Continuous variables were summarised using means and standard deviation (SD) or median, interquartile range (IQR), or range if subgroup was small. In univariate analysis, the difference between two groups was compared using chi-square test or Fisher's exact test, as appropriate. Continuous variables were analysed using a two-sample $t$ test or Mann-Whitney $U$ test if variables were not normally distributed. Odds ratio (OR) was calculated with $95 \%$ confidence interval (CI). Multivariate logistic regression was performed including variables that were clinically relevant, had univariate $p$ values less than 0.15 and were not multicollinear. $P$ value $<0.05$ was considered significant. SPSS version 22.0 (SPSS Inc., Chicago, IL, USA) was used for statistical analyses.

Factors associated with blood culture sampling were analysed also using multivariate logistic regression analysis and factors associated with bacteremia were analysed by comparing blood culture positive patients with blood culture negative patients. This approach differs from the analysis performed in the previous publications of this material $[14,15]$.

\section{Results}

\section{Blood culture findings}

In total, 460 patients with cSSSI were included. Blood cultures were drawn from 258 (51.6\%) patients and they were positive in $61(23.6 \%)$. Although there was some heterogeneity in the patient populations between the two centres [18], proportion of positive blood cultures was almost equal, in Helsinki $22.9 \%$ and $25 \%$ in Gothenburg. Blood culture isolates were Streptococcus pyogenes 19 cases (31.1\%), Staphylococcus aureus 19 (31.1\%), non-A beta-hemolytic streptococci 12 (19.7\%), Streptococcus pneumoniae 1 (1.6\%), Enterobacteriaceae spp. 1 (1.6\%), polymicrobial 5 $(8.2 \%)$ and unknown $(4 / 6.6 \%)$.

\section{Factors associated with blood culture sampling}

Patients from whom blood cultures were drawn $(n=258)$ were compared to those from whom blood cultures were not drawn $(n=202)$ for this analysis (Table 1$)$.

In logistic regression analysis, diabetes (OR 1.9, $P=$ $0.008)$, peripheral vascular disease (OR $0.5, P=0.007$ ), post-surgical wound infection (OR $0.4, P=0.005$ ), symptoms shorter than 2 days (OR 3.0, $P<0.001)$ and CRP over $150 \mathrm{mg} / \mathrm{L}$ on the first day (OR $1.8, P=0.006$ ) were significantly associated with blood culture sampling (Table 1).

\section{Factors associated with blood culture positivity}

To analyse factors associated with blood culture positivity, we compared patients with a positive blood culture $(n=61)$ to patients with a negative blood culture $(n=197)$.

Results of comparisons between the groups are shown in Table 2. Neither CRP measured at the time of the diagnosis of cSSSI nor the highest CRP during the hospital stay was associated with blood culture positivity (Table 2 ). In multivariate logistic regression analysis, only factor associated significantly with bacteremia was alcohol abuse (OR 5.5, P<0.001). 


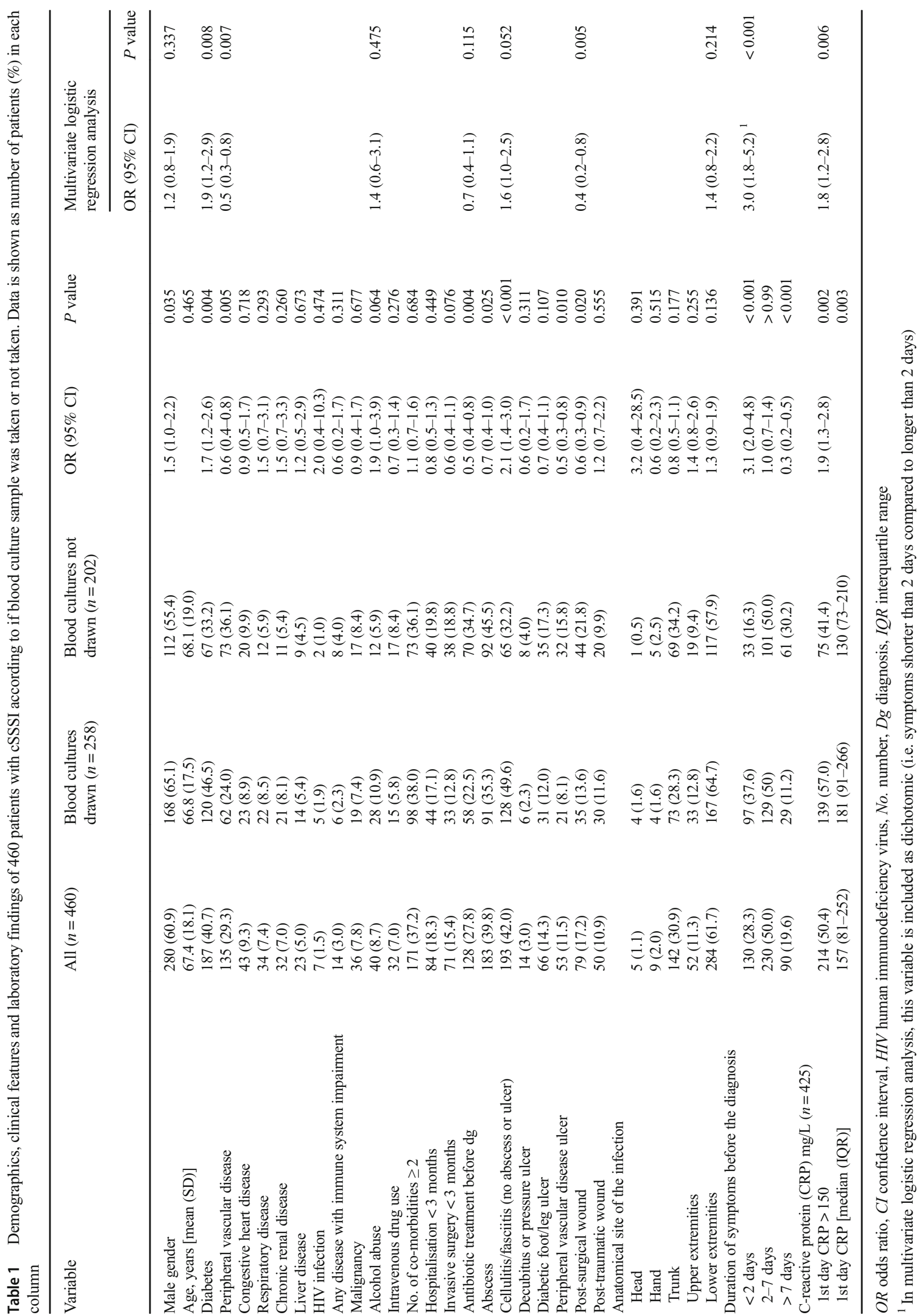




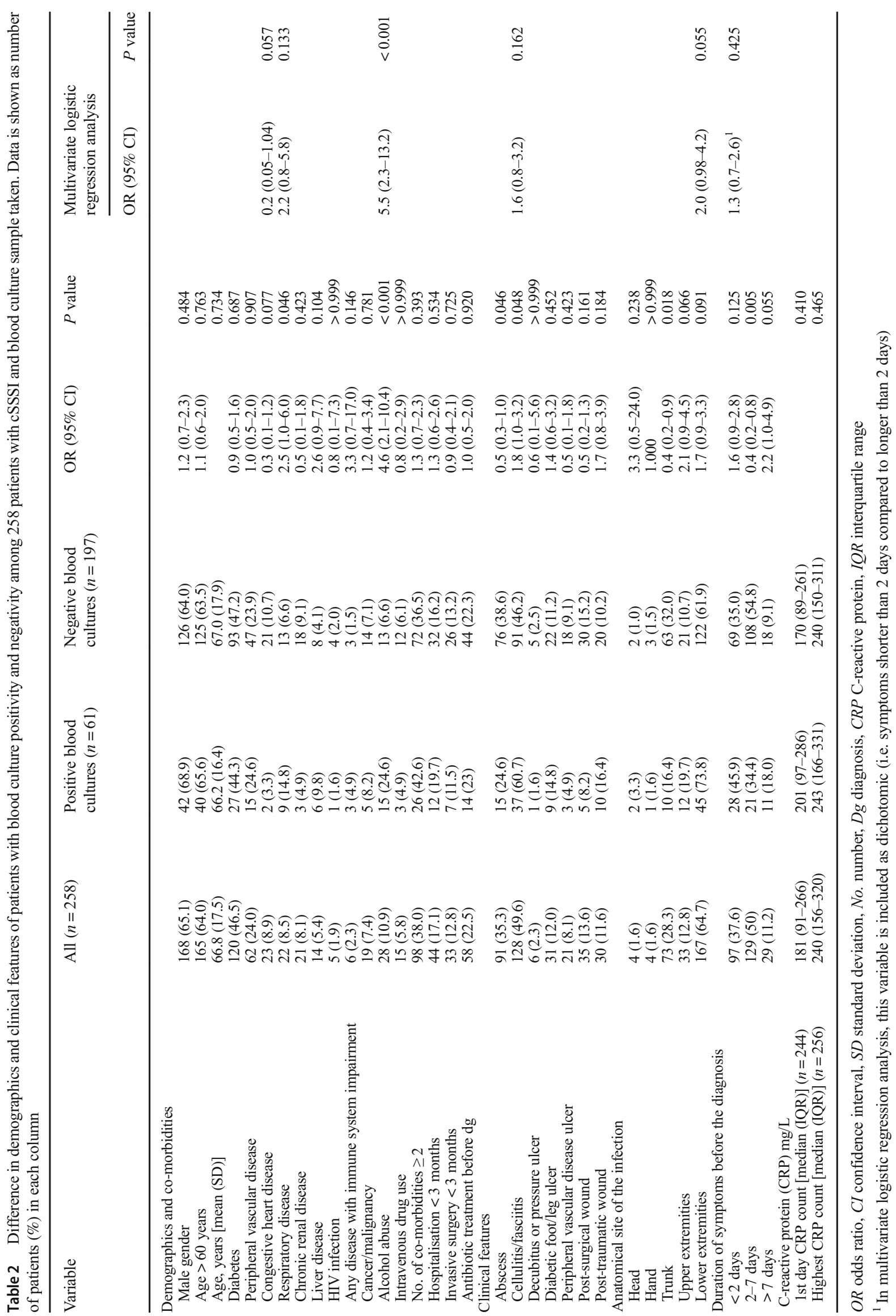




\section{Clinical endpoints in blood culture positivity}

Bacteremic patients $(n=61)$ were less likely to reach clinical stability within 3 days and they were more often admitted to intensive care unit and had significantly longer hospital stay than blood culture negative patients (Table 3 ). In addition, $23.3 \%$ of blood culture positive patients had antibiotic treatment streamlined as compared to $6.3 \%$ of culture negative patients $(P=0.0002)$.

\section{Discussion}

In this population-based study, we observed that $23.6 \%$ of the cSSSI patients from whom blood culture was taken had bacteremia. Streptococci and Staphylococcus aureus corresponded for $84 \%$ of cases. Bacteremia was associated with later clinical stability, more ICU admissions and more common streamlining. Higher CRP was linked to more common blood culture sampling, but not to culture positivity. Diabetes and duration of symptoms shorter than 2 days were observed to increase the likelihood of blood culture sampling, but only alcoholism increased the likelihood of blood culture positivity.

Blood culture positivity reported here was higher than $11.6 \%$ in a multi-centre study from Central and Southern Europe, which had a sampling rate of 53\% [11]. The most evident explanation is that our patient material was more severe. Blood culture positivity rate in less severe SSSIs like erysipelas or cellulitis has been reported to be 4.6-9\% [10,19].

In previous study on this patient material, it was observed that patients who had blood cultures drawn had higher mortality suggesting that clinicians ordered blood cultures from sicker patients [14]. Although the blood culture drawing rate differed in the two centres, the rate of positive findings was equal suggesting that blood cultures should most probably be taken with lower threshold in cSSSI.
Diabetes and higher CRP provoked clinicians to order blood culture sampling in accordance with data in erysipelas [19] and uncomplicated cellulitis [20]. In contrast to one previous study, prior antibiotic treatment was not negatively associated with positive blood culture findings but it resulted in less common (45.3\%) sampling than compared to patients without prior antibiotics $(54.7 \%, P=0.004)$ [21]. Alcohol abuse had a striking association with blood culture positivity, as of patients with a history of alcohol abuse $53.6 \%$ had bacteremia as compared to $20 \%$ of those who did not. Similarly, in patients with uncomplicated cellulitis, alcohol abuse was the only discriminant patient characteristic associated with bacteremia [20].

Beta-hemolytic streptococci and Staphylococcus aureus were not only the most common blood culture findings but they also constituted $64 \%$ of all cSSSI cases in which the aetiology was verified. Whereas we observed only one case of gram-negative monobacteremia, Van Daalen et al. found more gram-negative bacteria than Staphylococcus aureus [12] and Peralta et al. observed a gram-negative aetiology in $24.6 \%$ of bacteremias [21]. Accordingly, in a systematic review of patients with cellulitis and erysipelas, gram-negative bacteria were concluded to be at least as common as $S$. aureus in blood cultures in cellulitis [10]. These differences might be explained by difference in patient selection in these studies.

Our results contradict the view that blood cultures would not be useful because in complicated cellulitis they rarely had an effect on antibiotic therapy [9]. In our material, antibiotic treatment was streamlined more often in bacteremic than in non-bacteremic patients $(23.3 \%$ versus $6.3 \%, P<0.001)$. Accordingly, a change in the antibiotic treatment was recorded in $49 \%$ of patients with lower limb cellulitis after blood culture results became positive [21]. Furthermore, in countries with higher antibiotic resistance, the importance of blood culturedirected therapy has been pointed out [22].

Blood culture positivity was linked to later clinical stability, which without culture result might lead to a premature change of antibiotic treatment to more broad-spectrum. However,

Table 3 Outcome of 258 cSSSI patients from whom blood cultures were drawn. Data is shown as number of patients (\%) in each column

\begin{tabular}{|c|c|c|c|c|c|}
\hline Variable & All $(n=258)$ & $\begin{array}{l}\text { Positive blood } \\
\text { cultures }(n=61)\end{array}$ & $\begin{array}{l}\text { Negative blood } \\
\text { cultures }(n=197)\end{array}$ & $\begin{array}{l}\text { Odds ratio } \\
(95 \% \mathrm{CI})\end{array}$ & $P$ value \\
\hline Clinical stability within 3 days $(n=223)$ & $105(47.1)$ & $15(30)$ & $90(52)$ & $0.4(0.2-0.8)$ & 0.006 \\
\hline Admission to ICU & $51(19.8)$ & $20(32.8)$ & $31(15.7)$ & $2.6(1.4-5.0)$ & 0.003 \\
\hline Surgical intervention after the diagnosis of cSSSI & $129(50)$ & $34(55.7)$ & $95(48.2)$ & $1.4(0.8-2.4)$ & 0.305 \\
\hline 30-day mortality & $16(6.2)$ & $4(6.6)$ & $12(6.1)$ & $1.1(0.3-3.5)$ & $>0.999$ \\
\hline Streamlining $(n=251)$ & $26(10.4)$ & $14(23.3)$ & $12(6.3)$ & $4.5(2.0-10.5)$ & $<0.001$ \\
\hline $\begin{array}{l}\text { Duration of antimicrobial treatment, days } \\
{[\text { median (IQR)] }(n=255)}\end{array}$ & $21(12-38)$ & $26(11.5-46.8)$ & $20(12-38)$ & & 0.191 \\
\hline Length of hospital stay, days [median (IQR)] $(n=228)$ & $15(8-29)$ & $19.5(13-45.3)$ & $13(7-23)$ & & $<0.001$ \\
\hline
\end{tabular}

$C I$ confidence interval, IQR interquartile range 
positive blood culture was not linked to longer antibiotic treatment or higher mortality as has been reported previously [11]. The likely explanation to these differences is the low number of bacteremic patients and virtual lack of gram-negative and resistant bacteria in our study.

The strength of this study is its population-based nature, although some patients may have been unrecognised due to coding inaccuracy. Major limitations of this study are due to its retrospective nature. Data was collected from medical records, which left missing data in some parameters. Fifty-six percent of patients were subjected to blood culture sampling and were included in the analysis of factors associated with bacteremia, creating an inevitable selection bias.

In this population-based study in cSSSI, we observed that a positive blood culture was more common than previously reported and affected $23.6 \%$ of patients with blood culture sampled. Factors linked to higher blood culture sampling rate were not generally related to higher positive finding yield. A clear benefit of blood culture positivity on patient management was shown in more frequent antibiotic streamlining and knowledge of later clinical stability.

Acknowledgements We are grateful for biostatistician Tero Vahlberg for the statistical advice.

Funding Open access funding provided by University of Helsinki including Helsinki University Central Hospital. A clinical research grant is from Helsinki University Hospital. The data collection of this study was funded by AstraZeneca Nordic-Baltic.

\section{Compliance with ethical standards}

Conflict of interest MH has received a lecture fee from OrionPharma, Ratiopharm and MSD; a conference invitation from Gilead; and has recent consultancies with Pfizer. IHJ has received a conference invitation from Gilead. AJ has received speaker's honoraria from Astellas, Cardiome, MSD, OrionPharma, Pfizer, Ratiopharm and Unimedic; and congress support from MSD and Steripolar.

Ethical approval This study was approved by both study sites in local conventional manner and by the ethical committee of Sahlgrenska University Hospital.

Open Access This article is distributed under the terms of the Creative Commons Attribution 4.0 International License (http:// creativecommons.org/licenses/by/4.0/), which permits unrestricted use, distribution, and reproduction in any medium, provided you give appropriate credit to the original author(s) and the source, provide a link to the Creative Commons license, and indicate if changes were made.

\section{References}

1. Raff AB, Kroshinsky D (2016) Cellulitis: a review. JAMA 316: 325-337

2. Edelsberg J, Taneja C, Zervos M, Haque N, Moore C, Reyes K, Spalding J, Jiang J, Oster G (2009) Trends in US hospital admissions for skin and soft tissue infections. Emerg Infect Dis 15:1516-1518

3. Kaye KS, Patel DA, Stephens JM, Khachatryan A, Patel A, Johnson K (2015) Rising United States hospital admissions for acute bacterial skin and skin structure infections: recent trends and economic impact. PLoS One 10:e0143276

4. Center for Drug Evaluation and Research, Food and Drug Administration (1998) Guidance for industry: uncomplicated and complicated skin and skin structure infections - developing antimicrobial drugs for treatment. Washington, DC: Food and Drug Administration, US Department of Health and Human Services

5. Dryden MS (2010) Complicated skin and soft tissue infection. J Antimicrob Chemother 65(Suppl 3):iii35-iii44

6. Stevens DL, Bisno AL, Chambers HF, Dellinger EP, Goldstein EJ, Gorbach SL, Hirschmann JV, Kaplan SL, Montoya JG, Wade JC (2014) Infectious Diseases Society of America. Practice guidelines for the diagnosis and management of skin and soft tissue infections: 2014 update by the Infectious Diseases Society of America. Clin Infect Dis 59:e10-e52

7. Coburn B, Morris AM, Tomlinson G, Detsky AS (2012) Does this adult patient with suspected bacteremia require blood cultures? JAMA 308:502-511

8. Perl B, Gottehrer NP, Raveh D, Schlesinger Y, Rudensky B, Yinnon AM (1999) Cost-effectiveness of blood cultures for adult patients with cellulitis. Clin Infect Dis 29:1483-1488

9. Paolo WF, Poreda AR, Grant W, Scordino D, Wojcik S (2013) Blood culture results do not affect treatment in complicated cellulitis. J Emerg Med 45:163-167

10. Gunderson CG, Martinello RA (2012) A systematic review of bacteremias in cellulitis and erysipelas. J Inf Secur 64:148-155

11. Garau J, Ostermann H, Medina J, Avila M, McBride K, Blasi F, REACH study group (2013) Current management of patients hospitalized with complicated skin and soft tissue infections across Europe (2010-2011): assessment of clinical practice patterns and real-life effectiveness of antibiotics from the REACH study. Clin Microbiol Infect 19:E377-E385

12. van Daalen FV, Kallen MC, van den Bosch CMA, Hulscher MEJL, Geerlings SE, Prins JM (2017) Clinical condition and comorbidity as determinants for blood culture positivity in patients with skin and soft-tissue infections. Eur J Clin Microbiol Infect Dis 36:18531858

13. Lipsky BA, Kollef MH, Miller LG, Sun X, Johannes RS, Tabak YP (2010) Predicting bacteremia among patients hospitalized for skin and skin-structure infections: derivation and validation of a risk score. Infect Control Hosp Epidemiol 31:828-837

14. Jaaskelainen IH, Hagberg L, From J, Schyman T, Lehtola L, Jarvinen A (2016) Treatment of complicated skin and skin structure infections in areas with low incidence of antibiotic resistance-a retrospective population based study from Finland and Sweden. Clin Microbiol Infect 22:383.e1-383.e10

15. Jaaskelainen IH, Hagberg L, Forsblom E, Jarvinen A (2017) Factors associated with time to clinical stability in complicated skin and skin structure infections. Clin Microbiol Infect 23:674.e1674.e5

16. Jaakola S, Lyytikäinen $\mathrm{O}$, Rimhanen-Finne R, Salmenlinna S, Vuopio J, Roivainen M, Löflund J, Kuusi M, Ruutu P (2012) Tartuntataudit Suomessa 2011 [Infectious Diseases in Finland 2011]. Terveyden ja Hyvinvoinnin Laitos [National Institute for Health and Welfare]. Available at: http://urn.fi/URN:ISBN:978952-245-658-8

17. European Centre for Disease Prevention and Control (2012) Antimicrobial resistance surveillance in Europe 2011. Annual Report of the European Antimicrobial Resistance Surveillance Network (EARS-Net). ECDC, Stockholm

18. Jaaskelainen IH, Hagberg L, Schyman T, Jarvinen A (2018) A potential benefit from infectious disease specialist and stationary 
ward in rational antibiotic therapy of complicated skin and skin structure infections. Infect Dis (Lond) 50:107-116

19. Blackberg A, Trell K, Rasmussen M (2015) Erysipelas, a large retrospective study of aetiology and clinical presentation. BMC Infect Dis 15:402-015-1134-2

20. Bauer S, Aubert CE, Richli M, Chuard C (2016) Blood cultures in the evaluation of uncomplicated cellulitis. Eur J Intern Med 36:50-56

21. Peralta G, Padron E, Roiz MP, De Benito I, Garrido JC, Talledo F, Rodriguez-Lera MJ, Ansorena L, Sanchez MB (2006) Risk factors for bacteremia in patients with limb cellulitis. Eur J Clin Microbiol Infect Dis 25:619-626

22. Eron LJ, Lipsky BA (2006) Use of cultures in cellulitis: when, how, and why? Eur J Clin Microbiol Infect Dis 25:615-661

Publisher's note Springer Nature remains neutral with regard to jurisdictional claims in published maps and institutional affiliations. 